\title{
About buckling calculus of straight bars on elastic environment by Transfer-Matrix Method (TMM) for dental implants
}

\author{
Mihai Tripa ${ }^{1}$, Geamilia Şolea $^{2}$, Dorina Sorcoi $^{2}$, Iulia Florescu $^{2}$, Adriana Sorcoi $^{3}$, Daniela \\ Păunescu ${ }^{1}$, Nicolae Bâlc ${ }^{1}$ and Mihaela Suciu ${ }^{2 *}$ \\ ${ }^{1}$ Technical University of Cluj-Napoca, Machine Building Faculty, Bd. Muncii 103-105, Cluj- \\ Napoca, Romania \\ ${ }^{2}$ Technical University of Cluj-Napoca, Mechanical Faculty, Bd. Muncii 103-105, Cluj-Napoca, \\ Romania \\ ${ }^{3}$ Technical University of Cluj-Napoca, Materials and Environmental Engineering Faculty, Bd. \\ Muncii 103-105, Cluj-Napoca, Romania
}

\begin{abstract}
The paper presents a relatively simple and elegant analytical calculus of critical buckling force for a straight bar, one-end embedded and other end free, with an axial compression force $F$, using the TransferMatrix Method (TMM). The algorithm is based on the simplifications of the mathematical apparatus offered by Dirac and Heaviside's functions and operators regarding effort density. The results obtained will be used in the study of dental implants. The implant was assimilated as a bar on elastic environment, one-end of bar embedded and other end free, with an axial compression force $F$ at the free end, the bone being assimilated as an elastic environment.
\end{abstract}

\section{Introduction}

This study is the foundation for future research on titanium and its alloys of medicine domains, for prosthesis, in occurrence, in orthodontics, for dental implants. Medicine prosthesis are studies in $[1,2]$.

Due to mechanical properties similar to bone tissue, due to a good corrosion resistance and hardness, titanium and its alloys are most widely used.

For straight bars compressed with an axial force, risks of buckling are higher and knowing the critical buckling force is very important.

The classical calculus for critical buckling force is presented in [3].

We present a theoretical relatively simple analytical calculus of critical buckling force (after [4]) for a straight bar, one-end embedded and other end free, with an axial compression force $\mathrm{F}$ at the free end, using the Transfer-Matrix Method (TMM). The algorithm is based on the simplifications of the mathematical apparatus offered by Dirac and Heaviside's functions and operators regarding effort density, [4].

${ }^{*}$ Corresponding author: mihaela.suciu@rezi.utcluj.ro 
Results obtained will be used in studies for dental implants. The dental implant was assimilated as a bar on elastic environment, one-end of bar embedded and other end free, with an axial compression force $F$ at the free end, the bone being assimilated as an elastic environment.

Buckling of bio-composite sandwich bars are studied in [5] and bending beams on elastic environment by Transfer-Matrix Method (TMM) is studied in [6].

Approach for this problem of buckling bars on elastic environment is analyzed analytically by TMM, providing a validation basis for future experimental tests of titanium rods with different compositions, therefore with different material characteristics. Results will be also validated by Finite Element Method (FEM).

\section{Transfer-Matrix Method for a straight bar}

We will define the state vectors and transfer-matrix of a straight bar, loaded with a concentrated force on the ends (Figure 1.). In section $x$ we have the deformation $v$ [4].

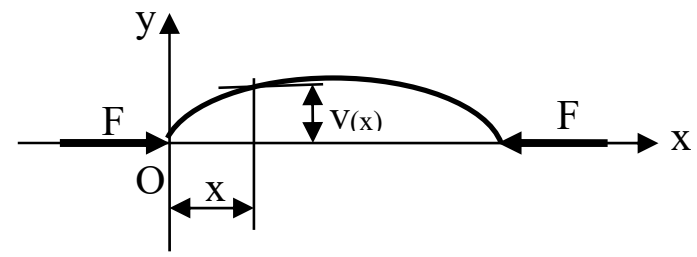

Fig. 1. Straight bar, loaded with a concentrated force on the ends.

\subsection{State vectors for a section}

We define the state vector for a section $x$ of a bar, a column-vector with 4 elements:

$$
\{U\}_{x}=\{U(x)\}=\{M(x), T(x), \omega(x), v(x)\}^{-1}
$$

For the origin, at $x=0$, we have the corresponding state vector:

$$
\{U\}_{0}=\left\{M_{0}, T_{0}, \omega_{0}, v_{0}\right\}^{-1}=\{U(0)\}=\{M(0), T(0), \omega(0), v(0)\}^{-1}
$$

And at the other end, for $x=l$, we have the corresponding state vector:

$$
\{U\}_{l 0}=\{U(l)\}=\{M(l), T(l), \omega(l), v(l)\}^{-1}
$$

\subsection{The Transfer Matrix for a straight bar}

We have the transfer-matrix $[\mathrm{T}]_{\mathrm{x}}$ for a bar between the origin and the section $x$ :

$$
\{U\}_{x}=[T]_{x}\{U\}_{0}+\left[T_{e}\right]_{x}\left\{U_{e}\right\}_{x}
$$

Where: $\{U\}_{x}$ - is the state vector associated of section $x$;

$[T]_{x}$ - is the transfer matrix between the origin and section $x$;

$\{U\}_{0}$ - is the state vector associated with the origin;

The term $\left[T_{e}\right]_{x} \cdot\left\{U_{e}\right\}_{x}$ - depends on the exterior loads corresponding at section $x$. 


\section{Differential equations of medium deformed fiber for a bar, which does not reveal on elastic environment}

We have a bar with length $l$, with constant moment of inertia over the entire length, on which a force $F$ acts in direction of axis of bar on extremities, which tend to compress the bar (Figure 2.).

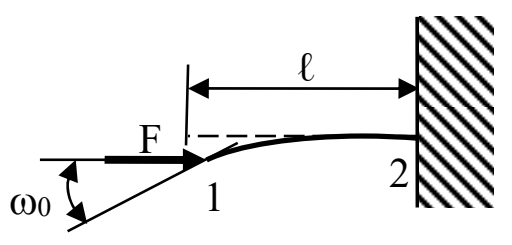

Fig. 2. Buckling bar, free at left end and embedded at right end.

Differential equation of medium deformed fiber is known [3]:

$$
E I \frac{d^{2} v}{d x^{2}}=-M(x)
$$

Where $M(x)$ is the total bending moment at the current point $x$, with the following condition: the curvature at the deformed elastic line is relatively small. Expression of bending moment at point $x$ is [4]:

$$
M(x)=-F\left[v(x)+C^{\prime \prime}\right]
$$

Constant $C^{\prime}$ takes into account that the origin arrow is different from 0 .

Differential equation of medium deformed fiber becomes successively:

$$
\begin{aligned}
& E I \frac{d^{3} v}{d x^{3}}=-F \frac{d v}{d x} \\
& E I \frac{d^{4} v}{d x^{4}}=-F \frac{d^{2} v}{d x^{2}}
\end{aligned}
$$

Or:

$$
E I \frac{d^{4} v}{d x^{4}}+F \frac{d^{2} v}{d x^{2}}=0
$$

(9) is a homogeneous linear differential equation with constant coefficients. If we note: $\alpha^{2}=\frac{F}{E I}$, equation (9) becomes:

$$
\frac{d^{4} v}{d x^{4}}+\alpha^{2} \frac{d^{2} v}{d x^{2}}=0
$$

With characteristic equation:

$$
r^{4}+\alpha^{2} r^{2}=0
$$


General solution of the homogeneous differential equation has the form:

$$
v(x)=A \cos \alpha x+B \sin \alpha x+C x+D
$$

Which will be derived four times, being obtained:

$$
\left\{\begin{array}{l}
v(x)=A \cos \alpha x+B \sin \alpha x+C x+D \\
\frac{d v}{d x}=-A \alpha \sin \alpha x+B \alpha \cos \alpha x+C \\
\frac{d^{2} v}{d x^{2}}=-A \alpha^{2} \cos \alpha x-B \alpha^{2} \sin \alpha x \\
\frac{d^{3} v}{d x^{3}}=A \alpha^{3} \sin \alpha x-B \alpha^{3} \cos \alpha x
\end{array}\right.
$$

The state vector in the origin is (2). With conditions in the origin, for $x=0$, the integration constants $A, B, C, D$ are obtained, as function of state vector in the origin:

$$
A=-\frac{M_{0}}{E I \alpha^{2}} ; \quad B=\frac{T_{0}}{E I \alpha^{3}} ; \quad C=\omega_{0}-\frac{T_{0}}{E I \alpha^{2}} ; \quad D=v_{0}+\frac{T_{0}}{E I \alpha^{2}}
$$

The solution of the homogeneous differential equation becomes:

$$
v(x)=v_{0}+\omega_{0} x+\frac{1-\cos \alpha x}{E I \alpha^{2}} M_{0}+\frac{\sin \alpha x-\alpha x}{E I \alpha^{3}} T_{0}
$$

\section{Transfer-Matrix for buckling bars}

By successively deriving for expression (15), we obtained:

$$
\left\{\begin{array}{l}
v(x)=v_{0}+\omega_{0} x+\frac{1-\cos \alpha x}{E I \alpha^{2}} M_{0}+\frac{\sin \alpha x-\alpha x}{E I \alpha^{3}} T_{0} \\
\omega(x)=\omega_{0}+\frac{\sin \alpha x}{E I \alpha} M_{0}+\frac{\cos \alpha x-1}{E I \alpha^{2}} T_{0} \\
M(x)=-\frac{\cos \alpha x}{E I} M_{0}+\frac{\sin \alpha x}{E I \alpha} T_{0} \\
T(x)=\frac{\alpha \sin \alpha x}{E I} M_{0}+\frac{\cos \alpha x}{E I} T_{0}
\end{array}\right.
$$

It is possible to write the matrix relation:

$$
\{U(x)\}=\left\{\begin{array}{l}
M(x) \\
T(x) \\
\omega(x) \\
v(x)
\end{array}\right\}=\left[\begin{array}{cccc}
-\frac{\cos \alpha x}{E I} & \frac{\sin \alpha x}{E I \alpha} & 0 & 0 \\
\frac{\alpha \sin \alpha x}{E I} & \frac{\cos \alpha x}{E I} & 0 & 0 \\
\frac{\sin \alpha x}{E I \alpha} & \frac{\cos \alpha x-1}{E I \alpha^{2}} & 1 & 0 \\
\frac{1-\cos \alpha x}{E I \alpha^{2}} & \frac{\sin \alpha x-\alpha x}{E I \alpha^{3}} & x & 1
\end{array}\right]\left\{\begin{array}{l}
M_{0} \\
T_{0} \\
\omega_{0} \\
v_{0}
\end{array}\right\}
$$

For $x=l,(17)$ becomes: 


$$
\{U(l)\}=\left\{\begin{array}{l}
M(l) \\
T(l) \\
\omega(l) \\
v(l)
\end{array}\right\}=\left[\begin{array}{cccc}
-\frac{\cos \alpha l}{E I} & \frac{\sin \alpha l}{E I \alpha} & 0 & 0 \\
\frac{\alpha \sin \alpha l}{E I} & \frac{\cos \alpha l}{E I} & 0 & 0 \\
\frac{\sin \alpha l}{E I \alpha} & \frac{\cos \alpha l-1}{E I \alpha^{2}} & 1 & 0 \\
\frac{1-\cos \alpha l}{E I \alpha^{2}} & \frac{\sin \alpha l-\alpha l}{E I \alpha^{3}} & l & 1
\end{array}\right]\left\{\begin{array}{l}
M_{0} \\
T_{0} \\
\omega_{0} \\
v_{0}
\end{array}\right\}
$$

The limit conditions are as follows:

$$
\left\{\begin{array}{l}
M_{l}=0 \\
T_{l}=F \omega_{0} \\
\omega_{l}=0 \\
v_{l}=0
\end{array}\right.
$$

And we can calculate the critical buckling force.

\section{Transfer-Matrix for buckling bars on elastic environment}

It is attempted to deduct the transfer matrix for a bar that is on a perfectly uniform and homogeneous elastic medium, knowing the function that is the coefficient of elasticity of the elastic medium $k(x)$, (Figure 3.).

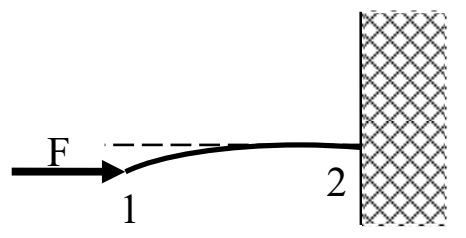

Fig. 3. Buckling bar, free at left end and on elastic environment at right end.

It is also assumed that the arrows at the two ends of the bar are initially 0 . The differential equation of the deformed average fiber is (3):

$$
E I \frac{d^{4} v}{d x^{4}}+F \frac{d^{2} v}{d x^{2}}+k v=0
$$

Which is a homogeneous linear differential equation of order 4. It is noted:

$\frac{k}{E I}=\varepsilon \quad \frac{F}{E I}=2 a$.

The characteristic equation is:

$$
r^{4}+2 a r^{2}+\varepsilon=0
$$

With the determinant:

$$
\Delta=a^{2}-\varepsilon
$$

For $a^{2}<\varepsilon$ does not exist solution. If $a^{2}>\varepsilon$ (after [4]), we can write: 


$$
\left\{\begin{array}{l}
\alpha^{2}=a+\sqrt{\Delta} \\
\beta^{2}=a-\sqrt{\Delta}
\end{array}\right.
$$

The general solution of differential equation (20) is:

$$
v(x)=A \cos \alpha x+B \sin \alpha x+C \cos \beta x+D \sin \beta x
$$

With constants:

$$
A=-\frac{1}{2 \sqrt{\Delta}}\left(\beta^{2} v_{0}+\frac{M_{0}}{E I}\right) ; B=-\frac{1}{2 \sqrt{\Delta}}\left(\beta^{2} \omega_{0}-\frac{T_{0}}{E I}\right) ; C=\frac{1}{2 \sqrt{\Delta}}\left(\alpha^{2} v_{0}+\frac{M_{0}}{E I}\right) ; D=v \frac{1}{2 \sqrt{\Delta}}\left(\alpha^{2} v_{0}+\frac{T_{0}}{E I}\right)
$$

That gives the general solution:

$$
v(x)=\frac{\alpha^{2} \cos \beta x-\beta^{2} \cos \alpha x}{2 \sqrt{\Delta}} v_{0}+\frac{\alpha^{3} \sin \beta x-\beta^{3} \sin \alpha x}{2 \alpha \beta \sqrt{\Delta}} \omega_{0}+\frac{\cos \beta x-\cos \alpha x}{2 E I \sqrt{\Delta}} M_{0}+\frac{\beta \sin \alpha x-\alpha \operatorname{sib} \beta x}{2 E I \alpha \beta \sqrt{\Delta}}
$$

Following the same steps as in $\S 4$, the matrix relationship (27) is obtained:

$$
\left\{\begin{array}{l}
M(x) \\
T(x) \\
\omega(x) \\
v(x)
\end{array}\right\}=\frac{1}{2 \sqrt{\Delta}}\left[\begin{array}{cccc}
\frac{\beta^{2} \cos \beta x-\alpha^{2} \cos \alpha x}{E I} & \frac{\alpha \sin \alpha x-\beta \sin \beta x}{E I} & \alpha \beta(\alpha \sin \beta x-\beta \sin \alpha x) & \alpha^{2} \beta^{2}(\cos \beta x-\cos \alpha x) \\
\frac{\alpha^{3} \sin \alpha x-\beta^{3} \sin \beta x}{E I} & \frac{\alpha^{2} \cos \alpha x-\beta^{2} \cos \beta x}{E I} & \alpha^{2} \beta^{2}(\cos \beta x-\cos \alpha x) & \alpha^{2} \beta^{2}(\alpha \sin \alpha x-\beta \sin \beta x) \\
\frac{\alpha \sin \alpha x \sin \beta x}{E I} & \frac{\cos \alpha x-\cos \beta x}{E I} & \alpha^{2} \cos \beta x-\beta^{2} \cos \alpha x & \alpha \beta(\beta \sin \alpha x-\alpha \sin \beta x) \\
\frac{\cos \beta x-\cos \alpha x}{E I} & \frac{\beta \sin \alpha x-\alpha \sin \beta}{E I \alpha \beta} & \frac{\alpha^{3} \sin \beta x-\beta^{3} \sin \alpha x}{\alpha \beta} & \alpha^{2} \cos \beta x-\beta^{2} \cos \alpha x
\end{array}\right]\left\{\begin{array}{l}
M_{0} \\
T_{0} \\
\omega_{0} \\
v_{0}
\end{array}\right\}
$$

\section{Conclusions}

The approach presented in this work will serve for o future research about dental implants. It is an original idea to assimilate the dental implant with a right bar on elastic environment loaded with a compressed force $F$. Calculus with Transfer-Matrix Method is very easy to program, for theoretical tests used to optimize the implants forms and after, in the future, to validate theoretical results with Finite Elements Method and experimental tests in laboratory and in situ.

\section{References}

1. P. Berce, N. Bâlc, C. Caizar, R. Păcurar, A. S. Radu, D. Brătean, I. Fodorean, Technology of manufacturing by adding material and their applications (Romanian Academy Publishing House, Bucharest, 2014), (in Romanian)

2. P. Berce, N. Bâlc, H. Chezan, D. Leordean, V. Mager, C. Borzan, C. Berce, Medical Applications of Manufacturing Technologies by Addition of Material (Romanian Academy Publishing House, Bucharest, 2015), (in Romanian)

3. C. Y. Warren, 1989, ROARC'S-Formulas for Stress \& Strain (6-ème Edition McGrawHill Book Company, 1989)

4. M. Gery, J.-A. Calgaro, Les Matrices-Transfert dans le calcul des structures (Editions Eyrolles, Paris, 1987)

5. M. Suciu, App. Mech. and Mat., 245, 39 (2013)

6. M. Suciu, App. Mech. and Mat., 186, 149 (2012) 